\title{
Stakeholders find that step therapy should be evidence-based, flexible, and transparent: assessing appropriateness using a consensus approach
}

\section{What is already known about this subject}

- Step therapy is a common, and growing, utilization management tool.

- Evidence of the effect of step therapy on health care costs and outcomes is mixed-the short-term cost savings may be outweighed by long-term increases in other health care use.

- Independent provider and patient groups have outlined guiding principles for step therapy protocols; legislation has been enacted in 24 states to minimize provider administrative burdens and to ensure patient protections when implementing step therapy.

\section{ABSTRACT}

BACKGROUND: Step therapy, one approach to utilization management, is used by health plans to ensure safe and clinically appropriate care while managing cost. Several patient and provider groups have each developed principles to guide the appropriate use of step therapy; however, no comprehensive multistakeholder informed set of criteria exist.

OBJECTIVE: To assess multistakeholder consensus on criteria for the development and implementation of step therapy for

\author{
What this study adds \\ - Using an iterative approach, \\ we achieved consensus on the \\ appropriateness of 21 criteria focused \\ on the development, implementation, \\ communication, and evaluation of \\ step therapy protocols among a \\ multistakeholder panel of 16 experts. \\ - Criteria related to the use of robust \\ clinical evidence when developing step \\ protocols, transparent protocols and \\ processes for patients and providers, \\ and appeals flexibility were rated as \\ standards (14/21) to which step therapy \\ policies should adhere to today. \\ - Criteria related to electronic tracking \\ of protocols and review processes, \\ as well as publicly sharing results \\ of protocol evaluations, were rated \\ as best practices $(7 / 21)$ that require \\ further research, policy solutions, or \\ infrastructure changes.
}

pharmaceutical therapies. Stakeholders were asked to (a) assess the appropriateness of step therapy as a utilization management tool; (b) rate specific criteria across 5 domains (development, implementation, communication, appeals, and evaluation) of step therapy; and (c) categorize these criteria as standards or best practices.

METHODS: We conducted a multiphase project culminating in a roundtable of experts representing patient, provider, plan, pharmacy, policy, and ethical perspectives. We first reviewed guiding principles,

\author{
Author affiliations \\ Taruja Karmarkar, PhD, MHS, Merck \& Co., \\ Kenilworth, NJ. Robert W Dubois, MD, PhD, \\ and Jennifer S Graff, PharmD, National \\ Pharmaceutical Council, Washington, DC. \\ AUTHOR CORRESPONDENCE: \\ Taruja Karmarkar, \\ taruja.karmarkar@merck.com \\ J Manag Care Spec Pharm. \\ 2021;27(2):268-75 \\ Copyright $\odot 2021$, Academy of Managed \\ Care Pharmacy. All rights reserved.
}

position statements, and legislative activity to draft criteria regarding step therapy protocol development, implementation, communication, and evaluation. To assess consensus across a convenience sample of experts, we employed an iterative 4-step modified Delphi method. Panelists were asked to (a) rate the overall appropriateness of step therapy, (b) rate the appropriateness of specific criteria, and (c) identify each as a standard or best practice. Appropriateness was rated from 1-9 and categorized in terciles (1-3: not appropriate, 4-6: neither, 
7-9: appropriate) to assess quantitative agreement, disagreement, and indeterminate agreement.

RESULTS: After the second round of voting, roundtable panelists $(n=16)$ disagreed on the appropriateness of step therapy for utilization management (50\% appropriate, $31.25 \%$ neither, and $18.75 \%$ inappropriate). Agreement was achieved on 21 criteria across 5 themes (clinical criteria as the foundation for protocol development, implementation of protocols, transparency and communication of processes, navigation of the appeals process, and evaluation of health and administrative impact). Fourteen and seven criteria were categorized as standards and best practices, respectively.

CONCLUSIONS: The stakeholders in this panel differed in their assessments of the appropriateness of step therapy but agreed regarding how these protocols should be developed, implemented, communicated, and evaluated. Most criteria were rated as standards that can be used by stakeholders when developing, implementing, and assessing step therapy processes today.

Utilization management tools used by payers, including step therapy (ST), allow for clinically appropriate, safe, and cost-effective patient care. When multiple treatments exist, patients are often required to try a clinically recognized firstline therapy before payment approval of more complex or expensive treatment options. In other scenarios, ST is used to manage treatments with safety, efficacy, or cost concerns.

Among employer-sponsored health plans, ST is common but varies in frequency of use and the number of steps in the protocol. ${ }^{1}$ Recently, the Centers for Medicare \& Medicaid Services (CMS) finalized a rule allowing Medicare Advantage plans to use ST in Part B drug coverage policies to increase competition and manage costs. ${ }^{2}$

Despite growing use of ST, the effect on health care costs and patient outcomes remains limited and mixed. ${ }^{3}$ ST encourages appropriate use of first-line drugs and has been shown to reduce drug spending in the short term for employer-sponsored and Medicare Part D plans and select therapeutic areas. ${ }^{4,5}$ However, it has also been shown to increase treatment discontinuation and medical resource use, such as emergency department visits..$^{5-8}$ The primarily observational and survey data are limiting factors to assessing the effect.

Physician and patient organizations have outlined ST principles that address physician administrative burden and potential unintended consequences. ${ }^{9-13}$ These groups emphasize flexibility to account for unique patient circumstances and timely and appropriate communication of protocols to patients. ${ }^{9,10}$ Several states have legislated patient protections for $\mathrm{ST}^{13,14}$
Stakeholder concerns and the growing use of ST, along with the uncertainty of its effect, underscores the need for standards when implementing ST protocols.

\section{Methods}

We reviewed the grey literature to identify guiding principles and position statements using the following search terms: ST principles, prior authorization guidelines, ST legislation, and utilization management guidelines. We searched legislative tracking tools for legislation regarding ST. ${ }^{13,14}$

Using these results, we drafted a checklist of criteria that encompassed common themes. We then engaged a subgroup of 6 experts representing patients, providers, plans, pharmacists, policy, and ethics perspectives to provide clarity to the criteria.

We used a 4-step modified Delphi method to assess consensus on the checklist criteria. ${ }^{15,16}$ This iterative method, often used in health care research, uses a systematic approach to collect and aggregate informed judgments from a group of well-informed experts on subjects where there is a lack of agreement, uncertainty, or lack of evidence. A roundtable was convened with 16 panelists, including the 6 experts previously mentioned, from the patient $(n=4)$, physician $(n=5)$, payer $(n=4)$, pharmacist $(n=1)$, health policy $(n=1)$, and ethics $(n=1)$ communities (see Acknowledgments). This convenience sample of experts was identified from the relevant peer-reviewed and grey literature or from their roles at relevant stakeholder organizations involved in ST processes.

The Delphi method included a premeeting round of voting, telephone interviews, and a group discussion followed by a second round of voting. During the roundtable, we provided panelists with their premeeting votes compared with the anonymized distribution of votes from the panel.

Assessing consensus was a 2-step process. We first asked panelists to rate the appropriateness of ST overall and the individual criterion on a scale from 1 (very inappropriate) to 9 (very appropriate). We then calculated median ratings and quantitative estimation of agreement or disagreement across 3 terciles (1-3: inappropriate, 4-6: neither, 7-9: appropriate).

Quantitative agreement (or disagreement) as determined by the modified Delphi calculation based on 16 panelists is determined by less (or more) than 4 responses at either end of the appropriateness terciles (Supplementary Figure 1, available in online article). For example, if 15 panelists voted a criterion as appropriate (7-9) and the remaining panelist rated it as inappropriate (1-3), we concluded agreement. If 5 panelists voted a criterion as inappropriate, 4 voted appropriate, and the remaining 7 felt it was neither (4-6), 
we would conclude disagreement. We concluded indeterminate agreement if neither criterion was met.

Criteria with a median appropriateness score of 7 or above or indeterminate agreement remained in the final checklist. By keeping indeterminate criteria in the checklist, the second level of differentiation, standards versus best practices, highlighted where the uncertainty remained. Our study aimed to capture those differences.

Panelists identified each criterion as a standard, a best practice, or neither. A criterion was a standard, a goal achievable today, if at least $75 \%$ of panelists rated it as a standard. If a criterion was rated by less than $75 \%$ of the panelists as a standard, but at least $75 \%$ of panelists rated it either a standard or a best practice, it was categorized as a best practice, or an aspirational goal that could be achieved through policy or infrastructure changes. We reported the final voting after round 2 .

\section{Results}

We identified 5 sets of recommendations from payer, provider, and patient organizations and 24 states with ST legislation. ${ }^{9-12,14}$ We found 5 common themes: (1) clinical criteria foundation for ST protocol development, (2) implementation of ST protocols, (3) transparency and communication of these protocols, (4) navigation of the appeals process, and (5) evaluation of the effect of ST. ${ }^{9-14}$ The subgroup provided clarity to checklist criteria (Table 1).

Overall, $50 \%$ of panelists rated ST as appropriate, while the other half were split between neither (31\%) and inappropriate (19\%; Figure 1). We concluded indeterminate agreement.

Several plan representatives highlighted the role of ST to ensure clinically appropriate medication use and improve patient health. Patient and provider representatives cautioned that ST is used to manage costs with few concerns regarding patient outcomes.

However, we found that panelists agreed on how ST should be implemented (Figure 1). At the conclusion of the roundtable, 21 criteria of the original 23 remained. Median scores across criteria ranged from 7 to 9; we found quantitative agreement or concluded indeterminate agreement among all checklist criteria.

Based on the $75 \%$ standard cut-off, the majority of the criteria $(14 / 21)$ were rated as standards, and 7 were best practices (Figure 1, also Supplementary Figure 2 and Supplementary Table 1, available in online article).

We summarize the final roundtable ratings, categorizations, and discussion to provide context.

\section{CLINICAL CRITERIA FOUNDATION FOR ST PROTOCOL DEVELOPMENT}

We found agreement on the appropriateness of 3 criteria related to ST protocol development. First, most panelists $(15 / 16)$ indicated that ST should be based on high-quality, up-to-date clinical evidence and prioritized before economic evidence. One plan representative noted their fiduciary responsibility and rated this as neither appropriate nor inappropriate.

Second, all panelists (16/16) agreed that ST protocols should be developed by an objective multidisciplinary review committee, free from potential conflicts of interest. However, panelists highlighted potential conflicts in integrated delivery systems where members of the committee are employed by the health plan.

Third, most panelists $(15 / 16)$ agreed that treatment failure, or risk of failure, should be defined using conditionspecific parameters. Patient and ethics representatives emphasized the importance of broad markers of failure such as loss of productivity. The group agreed that failure should include clinical metrics such as lack of efficacy and potential for adverse events, as well as additional concerns such as quality of life and provider judgment.

All 3 criteria were categorized as standards (Figure 1).

\section{IMPLEMENTATION OF ST PROTOCOLS AND PROCESSES}

We found quantitative agreement on the appropriateness of 5 of the 6 criteria associated with ST implementation and indeterminate agreement on 1 criterion.

First, all panelists (16/16) agreed that patients should face no more steps than clinically reasonable. However, determining what is "clinically reasonable" requires granular, timely, and unbiased clinical guidelines not always available.

Second, all panelists (16/16) concurred that trial duration should be specified for each treatment in the protocol, and time to "failure" should minimize patient harm. One panelist noted harm can extend beyond clinical measures if treatment affects a patient's quality of life or work status. Administrative burden should also be minimized.

Third, while many panelists (11/16) noted that responses to exception requests should occur rapidly, not all panelists rated this criterion as appropriate. Plan representatives explained that the response "clock" should begin once the plan has all documentation. In contrast, provider representatives emphasized the barriers to the seamless exchange of information across stakeholders due to lack of interoperability across systems.

Fourth, most panelists (13/16) agreed that once a plan authorizes a therapy, approval should remain as long as the 


\section{TABLE 1 Final Checklist Criteria}

Theme 1:

Clinical criteria

foundation for step

therapy protocol

development

A. Clinical evidence is considered before cost when reviewing medical products for coverage and potential for step therapy policy

B. Coverage policies, including step therapy, for medical products will be reviewed by an objective, external multidisciplinary committee and include the following inputs and processes:

- Member perspectives are considered in decision-making process

- All available clinical evidence, including studies on therapeutic need, efficacy, safety, and effectiveness

- Established clinical practice guidelines or compendia are incorporated into the review of clinical evidence

- Policies are updated regularly to reflect updated evidence for existing therapies

C. Failure, or risk of failure, is defined using condition-specific parameters (for lack of efficacy and adverse events) for any medical product when included in a step therapy protocol based on any of the following:

- Safety

- Surrogate endpoints and biomarkers

- Outcomes

- Health care provider determines drug is not in the best interest of the patient based on clinical judgment, medical necessity, or risk for treatment failure

\section{Theme 2:}

Implementation of step therapy protocols

A. Patients face no more steps than clinically reasonable and do not suffer undue burden in the treatment process

B. Time to "failure" in a step therapy protocol ensures patient does not face nor is at risk for harm, as demonstrated by the following:

- Health care provider clinical assessment of lack of treatment response

- Time limit for each step is specified in clinical criteria foundation

C. Request for exception is granted if benefits administrator does not respond to health care provider exception request in 72 hours or more rapidly for urgent situations

D. Once benefits administrator grants authorization, approval stands as long as the patient remains a beneficiary

E. Benefits administrator facilitates exceptions to step therapy policies to prevent interruptions in care, in the following circumstances:

- Patient has tried the required prescription drug while under their current or a previous health benefit plan, or another prescription drug in the same pharmacologic class or with the same mechanism of action

- Patient is currently stable on alternative therapy as determined by provider

- Treatment was not successful due to efficacy, tolerability, or safety

- If the benefits administrator has changed, prior step therapy results should be available for review

F. Completion of prior steps should be acceptable to subsequent plans

- Changes made to formulary should not interrupt treatment, during a current plan year, if patient is already on a previously approved therapy

- Benefits administrator should ensure appropriate grace period for patients if any changes to step edit protocol are implemented or altered

\section{Theme 3:}

Transparency of the processes and communication of protocols
A. Requirements for step therapy protocol, along with other formulary details, are easily accessible via a public link, in accessible language, on the benefits administrator's website

B. Benefits administrator communicates step edit protocols to both patient and health care provider

- Facilitate electronic exchange of information to reduce burden on providers

- Create standardized submission forms for exception or prior authorization requests

- Create a clear and accessible electronic process for contacting the benefits administrator

- Allow opportunity for patient or health care provider to make a request for an exception or appeal

C. Doctor: Electronic process for communication is implemented to allow patients and health care providers to view step edit protocol and exception status

D. Pharmacist: Electronic process for communication is implemented to allow patients and health care providers to view step edit protocol and exception status

E. Patient: Electronic process for communication is implemented to allow patients and health care providers to view step edit protocol and exception status

F. Any changes to formulary, including step edit protocols, will be transparent, in accessible language, to the patient and health care provider, and allow for a grace period following changes 


\section{TABLE 1 Final Checklist Criteria (continued)}

\begin{tabular}{|c|c|}
\hline $\begin{array}{l}\text { leme 4: } \\
\text { avigation of the } \\
\text { rceptions and } \\
\text { opeals processes }\end{array}$ & $\begin{array}{l}\text { A. Benefits administrator provides a clear, readily accessible, electronic process for health care provider and } \\
\text { patient to submit requests for appeals and exceptions, including the appeal request itself and supporting clinical } \\
\text { documentation (i.e., peer-reviewed medical literature, clinical guidelines, patient charts/history/test results) } \\
\text { B. Benefits administrator facilitates communication between prescribing health care provider and a provider of the } \\
\text { same training and specialty/subspecialty for discussion of medical necessity issues } \\
\text { C. If the exception or appeal is denied, health plan will provide relevant supporting documentation to applicant } \\
\text { for next steps including clinical justification for the decision, alternative options covered by the health plan and } \\
\text { process for requesting external review } \\
\text { D. Benefits administrator provides appeals approval statistics on health plan website for public review }\end{array}$ \\
\hline $\begin{array}{l}\text { Theme 5: } \\
\text { Evaluation of health } \\
\text { and administrative } \\
\text { impacts of step therapy }\end{array}$ & $\begin{array}{l}\text { A. Benefits administrator tracks processes and outcomes resulting from step therapy protocol implementation: } \\
\text { - Total annual step therapy requests, approvals, denials (aggregate and medication/indication level) } \\
\text { - Number and percentage of appeals accepted, denied (aggregate and medication/indication level) } \\
\text { - Report timing from submission of appeals documentation to approval or denial } \\
\text { - Response time to health care provider inquiry } \\
\text { - Rates of treatment discontinuation for drugs covered in step therapy protocol } \\
\text { - Days without treatment } \\
\text { - Trends in resource utilization over time to assess any unexpected change } \\
\text { B. Benefits administrator weighs administrative costs (i.e., staff time and resources) of reviewing step edits versus } \\
\text { costs to health plan to cover the prescribed medical product immediately }\end{array}$ \\
\hline
\end{tabular}

patient remains a beneficiary. However, plan representatives commented that changes might be warranted if there was (a) new evidence for an existing drug or (b) a newly approved therapy might be more appropriate. Most panelists agreed $(15 / 16)$ that plans should prevent interruptions in care by allowing exceptions to ST protocols if the patient is stable on treatment.

Finally, several panelists (11/16) felt that a patient's previously completed steps should be acceptable to subsequent plans. Improvements to electronic health information exchange should minimize administrative burden associated with switching plans. We concluded indeterminate agreement on this criterion.

One criterion-adherence to a time frame for response to exception requests-was identified as a best practice (Figure 1).

\section{TRANSPARENCY AND COMMUNICATION OF ST PROCESSES}

We found quantitative agreement on all 6 criteria regarding ST processes and protocol communications.

First, all panelists $(16 / 16)$ agreed that formulary details should be easily accessible on the plan website.

Second, most panelists (15/16) agreed that details should be communicated in an easily accessible manner to providers and patients.
Third, most panelists $(15 / 16)$ agreed it is appropriate to track exception or appeal requests electronically. Panelists identified the need for pharmacists to access this information given their role at the point of service; challenges persist because of e-prior authorization software limitations.

Most panelists (15/16) rated access to electronic tracking approval processes as an appropriate component of ST protocols. However, the group rated this a standard for doctors and a best practice for patients and for pharmacists. Panelists believed online portals could ensure patient privacy.

Finally, most panelists (15/16) agreed that communicating any formulary changes to patients in accessible language is necessary to keep them informed and ensure continuity of care.

Electronic tracking of ST protocol and review processes was the only criterion categorized as a best practice in this theme (Figure 1).

\section{NAVIGATION OF THE APPEALS PROCESS}

Criteria in this theme describe the steps to appeal a decision. We concluded agreement on 2 criteria and indeterminate agreement for the other 2 .

First, all panelists (16/16) agreed that the option to appeal a plan decision should be clear to the patient and the appeal submission process should be electronic and accessible to patients and providers. Panelists rated this a standard. 


\section{FIGURE 1 Round 2 Results: Appropriateness Ratings by Theme}

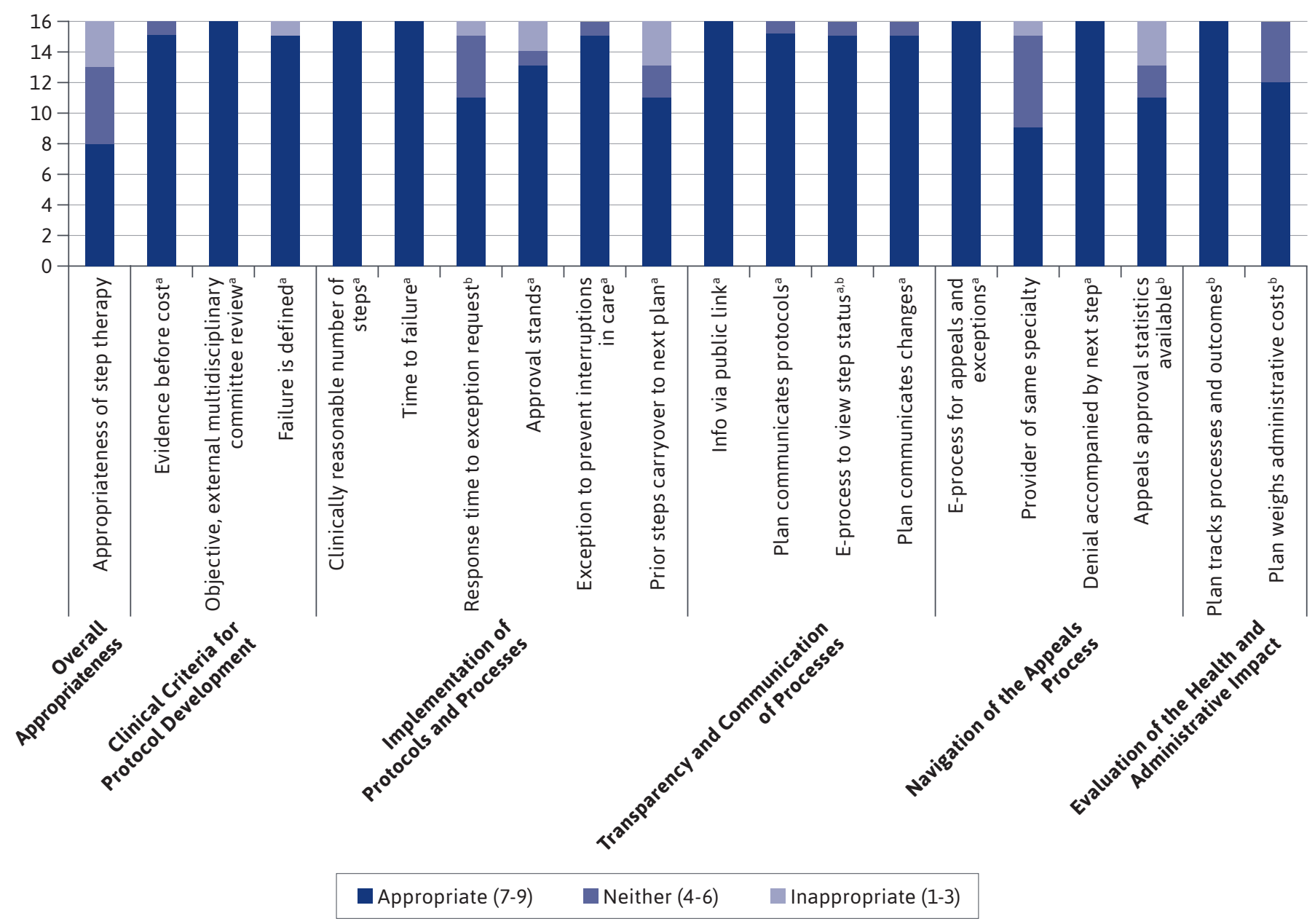

aStandard.

${ }^{b}$ Best practice.

Second, some panelists $(9 / 16)$ recognized that the need for peer-to-peer conversations during appeals review should include providers of similar specialties. Patient and provider representatives commented that straightforward requests could be addressed by general practitioners, but more complex situations may require providers of the same specialty. Further, 1 plan representative explained that the chief medical officer has final authorization regarding internal appeals; therefore, designating internal appeal requests to specialists may be difficult. This criterion was rated a best practice.

Third, all panelists (16/16) agreed, and rated as a standard, that if an appeal is denied, the plan should provide the decision rationale and the option for an external appeal and review to the provider and patient.

Fourth, panelists were split $(11 / 16)$ on whether it is appropriate for plans to make appeal statistics publicly available. Provider representatives advocated for appeals statistics to be available during plan enrollment. A plan representative noted this information is reported to CMS or state insurance review boards. However, plan representatives questioned who might access this information, what data should be available, whether measures are comparable, and the potential for misinterpretation. This criterion was rated a best practice. 


\section{EVALUATION OF HEALTH AND ADMINISTRATIVE IMPACT}

We found agreement on the appropriateness of 2 criteria related to the effect of ST.

First, all panelists (16/16) agreed that plans should track ST processes and outcomes (Figure 1). Plan representatives indicated that they track this information internally and, sometimes, report these data to accreditation bodies. While panelists agreed that documenting the results of a policy is needed, they voiced concerns with external reporting. Panelists rated this a best practice.

Second, most panelists $(12 / 16)$ believed it is appropriate for plans to weigh the administrative costs of implementing ST. One provider representative explained that any policy that affects a patient's access to care should be regularly evaluated. Others emphasized the administrative burden on physicians and pharmacists. However, as a plan representative noted, some ST protocols are implemented to prevent potential safety or abuse issues, so any benefits to patient safety should outweigh the administrative costs. The group agreed that these efforts to track and evaluate ST protocols were appropriate. However, differences in the need for public reporting likely led to the best practice categorization.

\section{Discussion}

A diverse group of stakeholders varied in their perspectives regarding the appropriateness of ST. This is not unexpected. Stakeholder benefits or concerns vary because of economics, care delivery, efficiency, and patient outcomes. ST protocols also vary in how they are implemented and then communicated to patients and providers across treatments and plans. Given these variations, the more important finding from our study was that stakeholders agreed on 21 criteria that plans should incorporate when developing and implementing ST programs. Most criteria were identified as standards for ST programs, while others were identified as best practices.

Previous work has focused on individual conditions, has been driven by 1 stakeholder perspective, or did not use a formal consensus process. The multistakeholder roundtable that we convened addressed issues related to ST that apply across therapeutic areas or patient populations. Further, the results from our approach to evaluate consensus are not dissimilar from those recommended by other professional bodies. ${ }^{17-19}$ Our panelists highlighted the importance of defining nuanced terms such as treatment "failure" and evaluating ST processes.
Decisions made by policymakers, through state and federal legislation or regulations, also affect how patients access care. Accreditation bodies and regulations, such as CMS Part D payment policies or state insurance requirements, are monitoring the use of ST. The results of our work might inform voluntary approaches such as accreditation or more transparent monitoring.

\section{LIMITATIONS}

There are limitations worth noting. First, we assessed consensus with a group of 16 experts representing patient, provider, and payer organizations-a different group may have different conclusions. Second, we found consensus on many of the checklist criteria in round 1 . The original criteria were developed while reviewing existing principles and legislation and refined through a small group before the roundtable-more controversial criteria may have been excluded. Third, ST policies are not implemented in a vacuum. Related but broader topics, such as prescription drug costs, were beyond the scope of this project.

\section{Conclusions}

Step therapy is a common and growing utilization management tool to encourage clinically appropriate care for patients and manage costs. While this panel expressed agreement on the approach to implementing ST, it also recognized that ST processes can create challenges for patients and providers. Existing and newly developed policies can and should adhere to common and agreed upon standards. Future research is needed to measure how well these criteria are adhered to today. In this way, we can ensure that ST, when used, promotes clinically appropriate care, mitigates patient and provider burden, ensures access to appropriate affordable care, and optimizes patient outcomes.

\section{DISCLOSURES}

This study was funded by the National Pharmaceutical Council. Karmarkar was a fellow at the National Pharmaceutical Council and Duke-Margolis Center for Health Policy at the time this study was conducted. Dubois and Graff are employees of the National Pharmaceutical Council.

This work was previously presented as a virtual poster during the AMCP 2020 eLearning Days, April 21-24, 2020. 


\section{ACKNOWLEDGMENTS}

The authors acknowledge the participation of the following panelists: Alan Balch, PhD (Patient Advocate Foundation); Lauren Bloch, MPA (Faegre Baker Daniels Consulting, SAIM Coalition); Jonathan Blum, MPP (Health Management Associates); Anne Burns, RPh (American Pharmacists Association); Joseph Cantrell, JD (American College of Rheumatology); Robert Carlson, MD (National Comprehensive Cancer Network); Eric Gascho (National Health Council); Estay Greene, PharmD (BlueCross BlueShield of North Carolina); Sharona Hoffman, JD (Case Western Reserve University); Jane Horvath, MS (Horvath Health Policy); Shawn Martin (American Academy of Family Physicians); Heather McComas, PharmD, MA (American Medical Association); Patrick Stone (National Psoriasis Foundation); Ronald Szabat, JD, LLM (American Academy of Child and Adolescent Psychiatry); Jeff White, PharmD, MS (Anthem); and 1 independent consultant who wished to remain anonymous. Some participants received travel reimbursement and honoraria; Cantrell, Carlson, Greene, McComas, and White did not accept honoraria. These acknowledgments do not imply endorsement of the criteria by the organizations listed.

\section{REFERENCES}

1. Chambers JD, Panzer AD, Neumann PJ. Variation in the use of step therapy protocols across US health plans. Health Affairs Blog, September 14, 2018. Accessed January 12, 2021. https:// www.healthaffairs.org/do/10.1377/ hblog20180912.391231/full/

2. Centers for Medicare \& Medicaid Services. Medicare Advantage and Part D Pricing Final Rule (CMS4180-F). CMS Newsroom. May 16, 2019. Accessed January 12, 2021. https:// www.cms.gov/newsroom/fact-sheets/ medicare-advantage-and-part-d-drugpricing-final-rule-cms-4180-f

3. Shoemaker J, Pozniak A, Subramanian R, Mauch D. Effect of 6 managed care pharmacy tools: a review of the literature. J Manag Care Pharm. 2010;16(Suppl 6-a):S1-S21. Accessed January 12, 2021. https://www.jmcp.org/doi/pdf/10.18553/ jmcp.2010.16.S6-A.1
4. Mark TL, Gibson TM, McGuigan K, et al. The effects of antidepressant step therapy protocols on pharmaceutical and medical utilization and expenditures. Am J Psychiatry. 2010;167(10):1202-09. doi: 10.1176/appi.ajp.2010.09060877

5. Shen X, Stuart BC, Powers CA, Tom SE, Magder LS, Perfetto EM. Impact of formulary restrictions on medication use and costs. Am J Manag Care. 2017;23(8):e265-e274. Accessed January 12, 2021. https://www.ajmc.com/ journals/issue/2017/2017-vol23-n8/ impact-of-formulary-restrictions-onmedication-use-and-costs

6. Stacey BR, Liss J, Behar R, et al. A systematic review of the effectiveness of policies restricting access to pregabalin. BMC Health Serv Res. 2017;17(1):600. doi: 10.1186/s12913-017-2503-x

7. Udall M, Louder A, Suehs BT, Cappelleri JC, Joshi AV, Patel NC. Impact of a step-therapy protocol for pregabalin on healthcare utilization and expenditures in a commercial population. J Med Econ. 2013;16(6):784-92. doi: 10.3111/13696998.2013.793692

8. Carlton RI, Bramley TJ, Nightengale B, Conner TM, Zacker C. Review of outcomes associated with formulary restrictions: focus on step therapy. Pharmacy Times. April 6, 2010. Accessed January 12, 2021. https://www.pharmacytimes.com/ publications/ajpb/2010/vol2_no1/reviewof-outcomes-associated-with-formularyrestrictions-focus-on-step-therapy

9. American Medical Association, et al. Prior authorization and utilization management reform principles. January 2017. Accessed January 12, 2021. https://www. ama-assn.org/system/files/2019-06/principles-with-signatory-page-for-slsc.pdf

10. American Society of Clinical Oncology. American Society of Clinical Oncology statement on the impact of utilization management policies for cancer drug therapies. J Oncol Pract. 2017;13(11):758-62. doi: 10.1200/JOP.2017.024273
11. National Arthritis Foundation. Position Statement on Step Therapy/ Fail First. September 2016. Accessed January 15, 2021. https://olis.leg. state.or.us/liz/2017R1/Downloads/ CommitteeMeetingDocument/98778

12. National Psoriasis Foundation. Position Statement on Access to Care. Revised September 2020. Accessed January 12, 2021. https://npf-website.cdn.prismic.io/ npf-website/e61c4c8d-a08c-4b38-9884de2c7e9af1b8_Position+Statement + on + Acc ess+to+Care+Revised+11.11.2020.pdf

13. SAIM Coalition. Legislation by state. Accessed January 12, 2021. https://www.saimcoalition.org/ saim-legislation-by-state

14. National Psoriasis Foundation. Step therapy legislation by state. Accessed January 12, 2021. http://www.steptherapy. com/step-therapy-legislation-by-state

15. Rand Corporation. Delphi method. Accessed January 12, 2021. https://www. rand.org/topics/delphi-method.html

16. McKenna H. The Delphi technique: a worthwhile research approach for nursing? J Adv Nurs. 1994;19(6):1221-25. doi: 10.1111/j.1365-2648.1994.tb01207.x

17. Robeznieks A. 1 in 4 doctors say prior authorization has led to a serious adverse event. American Medical Association. February 5, 2019. Accessed January 12, 2021. https://www.amaassn.org/practice-management/ sustainability/1-4-doctors-say-priorauthorization-has-led-serious-adverse

18. 2018-2019 Academy of Managed Care Pharmacy Professional Practice Committee. Prior Authorization and Utilization Management Concepts in Managed Care Pharmacy. J Manag Care Spec Pharm. 2019;25(6):641-44. doi: 10.18553/jmcp.2019.19069

19. AMCP Partnership Forum: optimizing prior authorization for appropriate medication selection. J Manag Care Spec Pharm. 2020;26(1):55-62. doi: 10.18553/ jmcp.2020.26.1.55 